\title{
Disruption of pulmonary lipid homeostasis drives cigarette smoke-induced lung inflammation in mice
}

\author{
Mathieu C. Morissette ${ }^{1}$, Pamela Shen ${ }^{2}$, Danya Thayaparan ${ }^{3}$ and \\ Martin R. Stämpfli ${ }^{1,4}$
}

Affiliations: ${ }^{1}$ Dept of Pathology and Molecular Medicine, McMaster Immunology Research Centre, McMaster University, Hamilton, ON, Canada. ${ }^{2}$ Medical Sciences Graduate Program, McMaster University, Hamilton, ON, Canada. ${ }^{3}$ Honours Molecular Biology and Genetics Co-op Program, McMaster University, Hamilton, ON Canada. ${ }^{4}$ Dept of Medicine, Firestone Institute of Respiratory Health at St Joseph's Healthcare, McMaster University, Hamilton, ON, Canada.

Correspondence: Martin R. Stämpfli, MDCL 4011, 1280 Main Street West, Hamilton, ON, L8S 4K1, Canada. E-mail: stampfliamcmaster.ca

ABSTRACT Overwhelming evidence links inflammation to the pathogenesis of smoking-related pulmonary diseases, especially chronic obstructive pulmonary disease (COPD). Despite an increased understanding of the disease pathogenesis, mechanisms initiating smoking-induced inflammatory processes remain incompletely understood.

To investigate the mechanisms that initiate and propagate smoke-induced inflammation, we used a wellcharacterised mouse model of cigarette smoke exposure, mice deficient for interleukin (IL)-1 $\alpha$, IL-1 $\beta$ and Toll-like receptor 4, and antibodies blocking granulocyte-macrophage colony-stimulating factor (GM-CSF). Studies were also pursued using intranasal delivery of human oxidised low-density lipoprotein (hOxLDL), a source of oxidised lipids, to investigate the inflammatory processes associated with impaired lipid homeostasis.

We found that cigarette smoke exposure rapidly led to lipid accumulation in pulmonary macrophages, a defining feature of foam cells, which in turn released high levels of IL- $1 \alpha$. In smoke-exposed IL- $1 \alpha$ deficient mice, phospholipids accumulated in the bronchoalveolar lavage, a phenomenon also observed when blocking GM-CSF. Intranasal administration of hOxLDL led to lipid accumulation in macrophages and initiated an inflammatory process that mirrored the characteristics of cigarette smoke-induced inflammation.

These findings identify a link between lipid accumulation in macrophages, inflammation and damaged surfactant, suggesting that the response to damaged pulmonary surfactant is a central mechanism that drives cigarette smoke-induced inflammation. Further investigations are required to explore the role of distorted lipid homeostasis in the pathogenesis of COPD.

@ERSpublications

Lipid accumulation in pulmonary macrophages drives cigarette smoke-induced lung inflammation http://ow.ly/MOLOU

Received: Nov 242014 | Accepted after revision: April 072015 | First published online: June 252015

Support statement: This study was funded by the Flight Attendant Medical Research Institute (grant number 123386) and the Canadian Institutes of Health Research (Government of Canada; grants MOP-123369 and MPO-64390). Funding information for this article has been deposited with FundRef.

Conflict of interest: Disclosures can be found alongside the online version of this article at erj.ersjournals.com

Copyright $\odot$ ERS 2015 


\section{Introduction}

The adverse health consequences of smoking are numerous; smoking is a major risk factor for chronic obstructive pulmonary disease (COPD), interstitial lung diseases and lung cancer, in addition to increasing susceptibility to microbial infections [1]. All these conditions are strongly associated with smoke's impact on the immune system and the initiation of a chronic inflammatory state [1]. While characteristics of cigarette smoke-induced inflammation have been investigated for decades, mechanisms that ultimately initiate and drive these processes remain poorly understood.

One of the most striking features associated with smoking is the increase in size and complexity of the pulmonary macrophage [2,3], a critical immune sentinel [4] and key player in the catabolism of damaged surfactant [5]. Cigarette smoke's damaging effect on surfactant lipids was initially proposed in the late 1960s and 1970s [3, 6]. Moreover, our group recently observed the conserved induction of genes involved in lipid metabolism by cigarette smoke in the lungs of both humans and mice [7]. While there is clear evidence that smoking leads to lipid oxidation in the lungs [8-10], the relevance of these observations to inflammatory processes associated with smoking has not been investigated. We built on these findings and studied the relationship between pulmonary surfactant homeostasis and mechanisms of cigarette smoke-induced inflammatory processes.

We found that cigarette smoke exposure rapidly led to lipid accumulation in pulmonary macrophages, an important characteristic of foam cells. Lipid accumulation in macrophages led to the production of interleukin (IL)-1 $\alpha$, probably a protective response to restore lipid homeostasis, as phospholipids rapidly accumulated in the bronchoalveolar lavage (BAL) of mice deficient in IL- $1 \alpha$, or when blocking granulocyte-macrophage colony-stimulating factor (GM-CSF). Notably, administration of oxidised lipids intranasally mimicked cellular and molecular inflammatory processes elicited by smoke exposure. Overall, our findings suggest that smoke-induced inflammatory processes are triggered by damage to pulmonary lipids.

\section{Materials and methods}

Mice

$\mathrm{BALB} / \mathrm{c}, \mathrm{C} 57 \mathrm{BL} / 6$ (wild type control for IL- $1 \alpha^{-/-}$and IL-1$\beta^{-/-}$mice), C3H/HeOuJ (wild type for Toll-like receptor 4 (TLR4)) and $\mathrm{C} 3 \mathrm{H} / \mathrm{HeJ}$ (defective for TLR4) mice were purchased from the Jackson Laboratory (Bar Harbor, ME, USA). IL- $1 \alpha^{-/-}$and IL-1 $\beta^{-/-}$mice were a kind gift from Yoichiro Iwakura (University of Tokyo, Tokyo, Japan). Mice were housed according to the Canadian Council for Animal Care guidelines and McMaster University's Animal Research Ethics Board approved all procedures.

\section{Cigarette smoke exposure}

Mice were exposed to mainstream smoke of 3R4F reference cigarettes (University of Kentucky, Lexington, KY, USA) with filters removed, using a whole-body exposure system (SIU48; Promech Lab Holding AB, Vintrie, Sweden) as described in detail previously [11, 12]. Briefly, mice were exposed twice daily to mainstream cigarette smoke from 12 cigarettes. Total particulate matter ranged from 700 to $900 \mu \mathrm{g} \cdot \mathrm{L}^{-1}$. We previously showed that cotinine and carboxyhaemoglobin levels in cigarette smoke-exposed mice are comparable to those observed in human smokers [11].

\section{Human oxidised low-density lipoprotein and GM-CSF-neutralising antibody delivery}

For intranasal delivery of human oxidised low-density lipoprotein (hOxLDL), C57BL/6 mice were anaesthetised with isoflurane. Three consecutive intranasal deliveries of $70 \mu \mathrm{g}$ (total protein content) of hOxLDL (\#BT-910; Biomedical Technologies Inc., Ward Hill, MA, USA) in $35 \mu \mathrm{L}$ of PBS were delivered $\sim 12 \mathrm{~h}$ apart starting on the evening of the first day. Mice were sacrificed $12 \mathrm{~h}$ after the last hOxLDL delivery. Control mice were administered PBS only.

To deliver the GM-CSF-neutralising antibody, C57BL/6 mice were anaesthetised with isoflurane. $20 \mu \mathrm{g}$ of GM-CSF-neutralising or isotype control antibodies (clone MP1-22E9 and RTK2758; Biolegend, San Diego, CA, USA) were delivered intranasally in $35 \mu \mathrm{L}$ of PBS daily prior to the first cigarette smoke exposure.

\section{BAL collection and assessment}

Mice were anaesthetised with isoflurane. The lungs were removed and the tracheae were cannulated and lavaged twice with $500 \mu \mathrm{L}$ of cold PBS. BAL total cell numbers were determined using a haemocytometer. Cells were pelleted and the cell-free BAL fluid (BALF) was collected. BALF was stored at $-80^{\circ} \mathrm{C}$ until further analyses. Cytospins were prepared following PBS resuspension of cells and stained using Hema3 (Fisher Scientific Company, Ottawa, ON, Canada). 300 cells were counted per cytospin. Levels of IL-1 $\alpha$, GM-CSF and phosphatidylcholine in the BALF were measured according to the manufacturers' instructions (mouse IL- $1 \alpha$ Duoset ELISA: R\&D Systems, Minneapolis, MN, USA; mouse GM-CSF ELISA 
Ready-Set-Go: eBioscience, San Diego, CA, USA; phosphatidylcholine colorimetric assay kit: Cayman Chemical, Ann Arbor, MI, USA).

\section{Pulmonary macrophage size determination}

The size of pulmonary macrophages was determined using the ImageJ Software (v1.44o; ImageJ, http:// imagej.nih.gov/ij). 35 macrophages per cytospin per mouse were randomly picked and the surface area measured. Lymphocytes (bright blue nucleus and small cytoplasm) were excluded from the analysis. The mean size of the pulmonary macrophages from mice exposed to room air was set as $100 \%$. The $150 \%$ value represents the 95 th percentile and $300 \%$ represents the 95 th percentile multiplied by two.

\section{BODIPY staining and visualisation}

BAL cell cytospins were fixed in $10 \%$ formalin for $15 \mathrm{~min}$ and washed with PBS three times. Cytospins were stained using a solution of BODIPY (boron-dipyrromethene) 493/503 and DAPI (4',6-diamidino-2-phenylindole) in PBS (2.5 and $5 \mu \mathrm{g} \cdot \mathrm{mL}^{-1}$, respectively; both from Life Technologies Inc., Burlington, ON, Canada). After two PBS washes, slides were mounted in PBS and fluorescence was acquired using a Leica DMRA microscope (Leica Microsystems GmbH, Wetzlar, Germany).

\section{Transmission electron microscopy}

Pieces of unlavaged lung tissue were fixed overnight ( $2 \%$ glutaraldehyde (v/v) in $0.1 \mathrm{M}$ sodium cacodylate, $\mathrm{pH}$ 7.4). Samples were rinsed twice in buffer solution, post-fixed in $1 \%$ osmium tetroxide in $0.1 \mathrm{M}$ sodium cacodylate for $1 \mathrm{~h}$ and then dehydrated through a graded ethanol series $(50 \%$, twice in $70 \%$, twice in $95 \%$, twice in 100\%). Final dehydration was performed in two changes of $100 \%$ propylene oxide. The tissue pieces were slowly infiltrated with Spurr's resin through a graded series (propylene oxide/Spurr's ratios of $2: 1,1: 1$ then $1: 2$, then three times in $100 \%$ Spurr's) with rotation of the samples between solution changes. The samples were then transferred to embedding moulds filled with fresh $100 \%$ Spurr's resin and polymerised overnight at $60^{\circ} \mathrm{C}$. Thin sections were cut on a Leica UCT ultramicrotome (Leica Microsystems $\mathrm{GmbH}$ ) and placed on copper grids. Sections were post-stained with uranyl acetate and lead citrate and viewed in a JEOL JEM 1200 EX TEMSCAN transmission electron microscope (JEOL, Peabody, MA, USA) operating at an accelerating voltage of $80 \mathrm{kV}$. Images were acquired with an AMT 4-megapixel digital camera (Advanced Microscopy Techniques, Woburn, MA, USA).

\section{Statistical analysis}

Two-group comparisons were performed using unpaired t-tests. Multiple-group comparisons were performed using a one-way ANOVA followed, if applicable $(\mathrm{p}<0.05)$, by a Bonferroni's multiple comparisons test. Tests were performed using Prism 5 from GraphPad Software, Inc. (La Jolla, CA, USA).

\section{Results}

Cigarette smoke exposure leads to lipid accumulation in pulmonary macrophages

Using a mouse model, we analysed the size of pulmonary macrophages after defined periods of cigarette smoke exposure and investigated the cause for their increase in size. Similar to what is observed clinically, pulmonary macrophages in the BAL of cigarette smoke-exposed $\mathrm{BALB} / \mathrm{c}$ mice progressively increased in size (fig. 1a). The number of macrophages that were size $>300 \%$ (more than twice the 95th percentile of the size of the pulmonary macrophages from room air-exposed mice) increased by four-fold between weeks 1 and 8 of smoke exposure. This number further doubled after exposure for 24 weeks and remained elevated following smoking cessation for 60 days (fig. 1b). This increase in size was associated with a high prevalence of vesicle-like structures within the cytoplasm (fig. 1c), giving them the appearance of foam cells, lipid-laden macrophages described in atherosclerosis [13, 14]. Staining with the lipophilic dye BODIPY revealed that pulmonary macrophages from smoke-exposed mice were filled with lipid droplets (fig. 1d). We further visualised the lipid droplets by transmission electron microscopy (fig. 1e). These findings suggest that the progressive increase in size of pulmonary macrophages following cigarette smoke exposure is caused, at least in part, by the accumulation of lipids, a characteristic feature of foam cells.

\section{IL-1a release in response to smoking is crucial to maintain pulmonary lipid homeostasis}

We next investigated the contribution of lipid-laden macrophages to inflammatory processes associated with smoking. To this end, $\mathrm{BALB} / \mathrm{c}$ mice were exposed to cigarette smoke for 4 days. Pulmonary macrophages isolated from cigarette smoke-exposed mice expressed high levels of IL-1 $\alpha$ (fig. 2a). To assess the importance of IL- $1 \alpha$, we exposed IL- $1 \alpha$-deficient, as well as wild-type control and IL-1 $\beta$-deficient mice, to cigarette smoke for 4 days. Counting mononuclear cells and neutrophils showed that IL-1 $\alpha$ is a critical mediator controlling inflammatory processes associated with smoke exposure (fig. $2 b$ and $c$ ) $[12,15]$. In addition to absence of cellular inflammation, we observed that the cell-free BALF of cigarette smoke-exposed IL-1 $\alpha$-deficient mice had a cloudy appearance (fig. $2 \mathrm{~d}$ ), measurable by light scattering at 

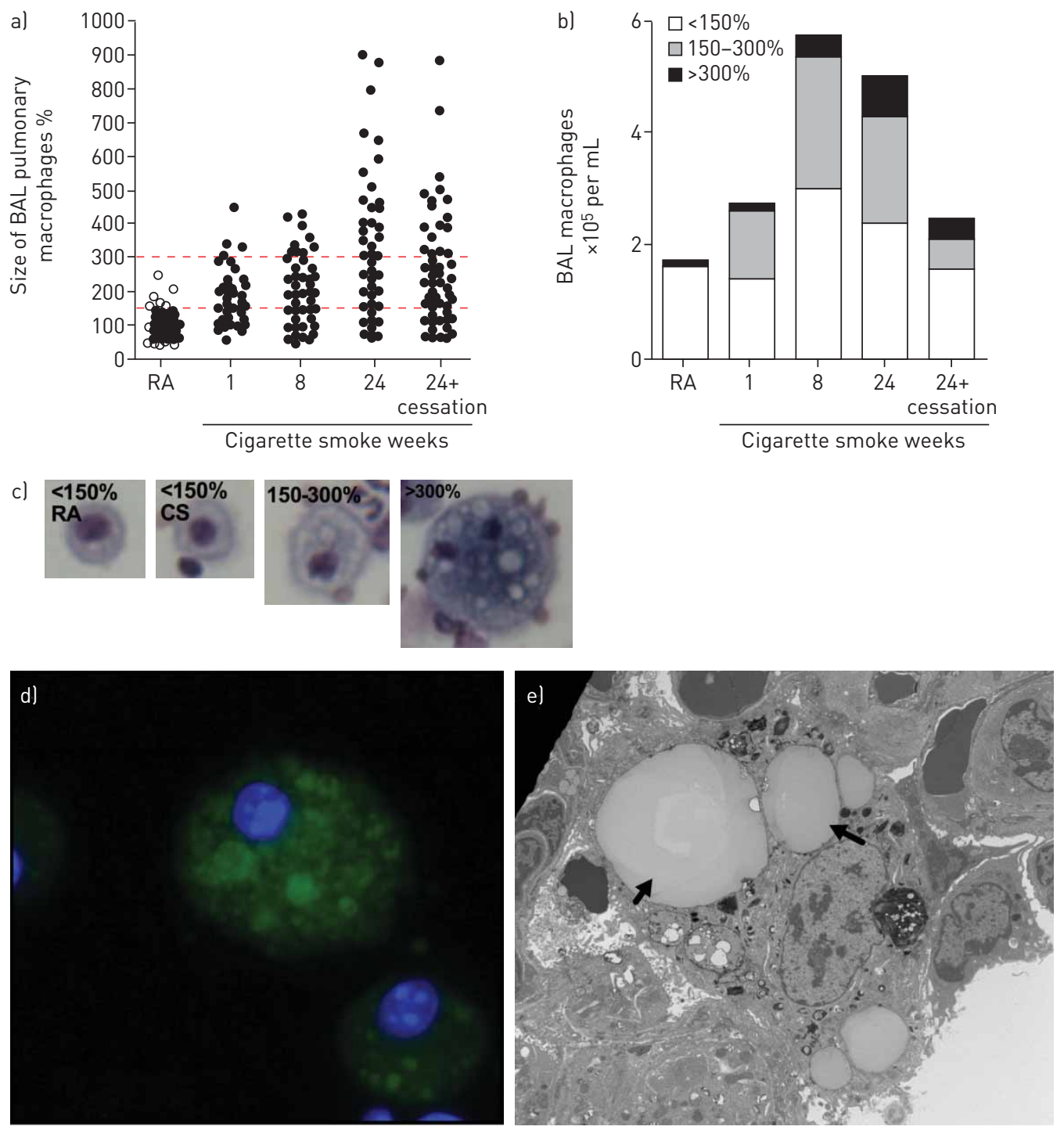

FIGURE 1 Cigarette smoke exposure leads to lipid accumulation in pulmonary macrophages. a and b) Size distributions of pulmonary macrophages from the bronchoalveolar lavage (BAL) of BALB/c mice exposed to room air (RA) and cigarette smoke (CS), and following smoking cessation (five mice per group). The mean size of the pulmonary macrophages from RA-exposed mice was set as $100 \%$. The $150 \%$ value represents the 95 th percentile and $300 \%$ represents the 95th percentile multiplied by two. c) Representative images (haematoxylin and eosin staining) of the different size and exposure groups. Neutral lipid accumulation in pulmonary macrophages from CS-exposed mice was shown by d) BODIPY (boron-dipyrromethene; green) incorporation (with DAPI (4',6-diamidino-2-phenylindole; blue) staining of nuclei) and e) transmission electron microscopy. Arrows show lipid droplets.

$550 \mathrm{~nm}$ (fig. 2e). This increase in cloudiness was attributable to a marked increase in BALF total phosphatidylcholine (fig. 2f), which represents the majority lipid species of the pulmonary surfactant lipid fraction [16]. Based on these findings, we postulate that IL-1 $\alpha$-dependent inflammation following smoke exposure is required to maintain surfactant homeostasis in the lungs.

\section{Production of GM-CSF in response to IL-1 $\alpha$ is necessary to maintain lipid homeostasis}

Clinical and experimental evidence has demonstrated that GM-CSF plays a critical role in surfactant homeostasis [5]. We therefore investigated the importance of GM-CSF in the maintenance of surfactant homeostasis following smoke exposure. C57BL/ 6 wild type, IL-1 $\alpha$-deficient and IL-1 $\beta$-deficient mice were exposed to room air or cigarette smoke for 4 days. As previously reported [12, 17], we observed increased GM-CSF expression following cigarette smoke exposure (fig. 3a). Moreover, GM-CSF induction was dependent on IL-1 $\alpha$ and partly on IL-1 $\beta$ (fig. 3a). Interference with GM-CSF signalling using monoclonal antibodies led to an increase in BALF cloudiness (fig. 3b), absorbance (fig. 3c) and total phosphatidylcholine content (fig. 3d) in C57BL/6 mice exposed to room air or cigarette smoke for 4 days. This increase was 

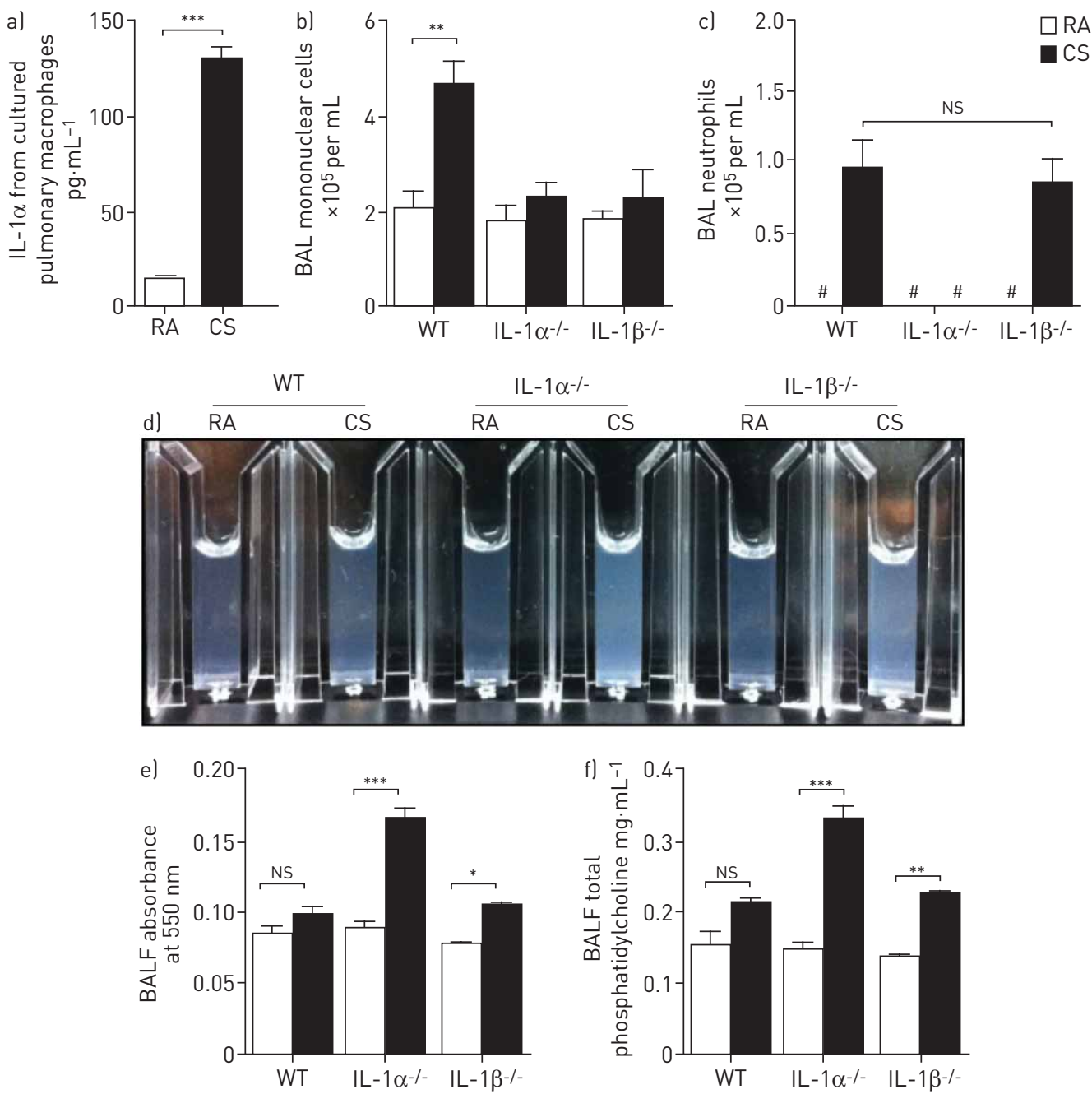

FIGURE 2 Interleukin (IL)-1 $\alpha$-mediated immune response is required to maintain surfactant homeostasis following cigarette smoke (CS) exposure. a) Ex vivo release of IL-1 $\alpha$ by pulmonary macrophages from BALB/c mice exposed to room air (RA) or CS for 4 days. b-f) C57BL/6 wild type (WT), IL-1 $\alpha$-deficient and IL-1 $\beta$-deficient mice were exposed to CS for 4 days. CS induced increases in bronchoalveolar lavage (BAL) b) mononuclear cells and c) neutrophils. d) Representative cell-free BAL fluid (BALF). e) Quantification of BALF cloudiness by absorbance measurement at $550 \mathrm{~nm}$. f) BALF phosphatidylcholine levels. At least five animals per experimental group, reproduced at least twice. Ns: nonsignificant. ${ }^{\#}$ : not detected. ${ }^{*}: \mathrm{p}<0.05 ;{ }^{* *}: \mathrm{p}<0.01 ;{ }^{* *}: \mathrm{p}<0.001$.

observed in cigarette smoke-exposed mice despite the normal increase in mononuclear cells and neutrophils (fig. $3 \mathrm{e}$ and $\mathrm{f}$ ) and in IL-1 $\alpha$ levels (fig. 3g). These findings suggest a novel role for IL-1 $\alpha$ in maintaining surfactant homeostasis through the induction of GM-CSF, probably by activating mechanisms involved in surfactant degradation by immune cells.

\section{Pulmonary administration of oxidised lipids mimics cigarette smoke-induced inflammation}

So far, our findings strongly suggest that alterations in surfactant homeostasis drive the inflammatory process triggered by smoking. As a proof of concept, and in an attempt to mimic the increased pressure on the lipid catabolism machinery caused by smoking, we administered hOxLDL to force the lung to process a large amount of damaged lipids. hOxLDL was delivered intranasally into anaesthetised C57BL/6 mice on three consecutive occasions, $12 \mathrm{~h}$ apart, as detailed in figure $4 \mathrm{a}$. The cellular profile and the appearance of pulmonary macrophages recovered from the lungs of mice that received hOxLDL intranasally were similar to mice exposed to cigarette smoke (fig. 4b). Intranasal hOxLDL administration, like cigarette smoke exposure, caused intracellular lipid accumulation (fig. $4 \mathrm{c}$ and d), as well as enlargement of pulmonary macrophages (fig. 4e). As is the case with cigarette smoke exposure, intranasal hOxLDL delivery induced the release of IL-1 $\alpha$ (fig. $4 \mathrm{f}$ ), neutrophil recruitment (fig. 4g) and GM-CSF production (fig. $4 \mathrm{~h}$ ). Using IL-1 $\alpha$-deficient and IL-1 $\beta$-deficient mice, we further showed that hOxLDL-associated neutrophil 

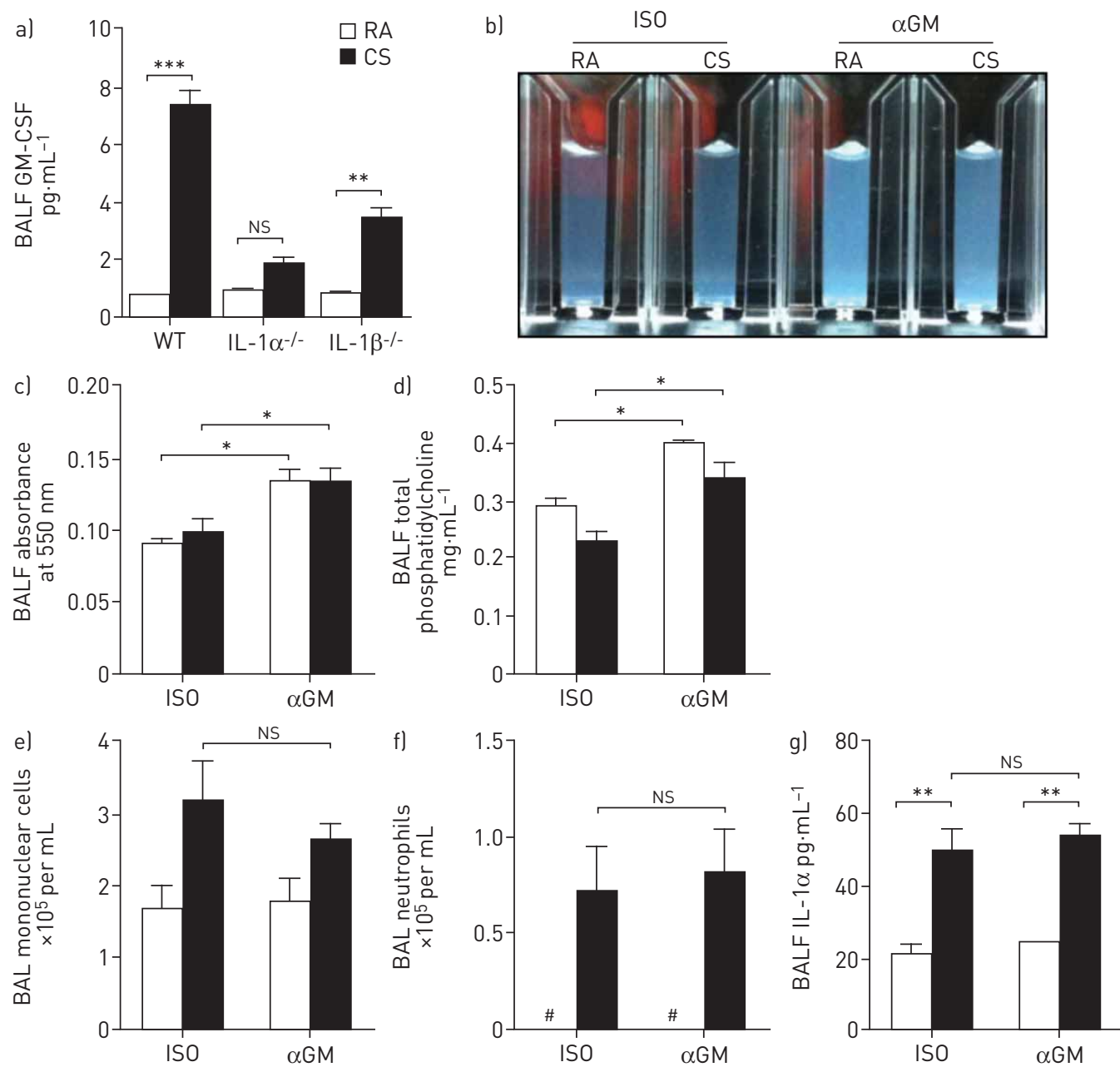

FIGURE 3 Interleukin (IL)-1 $\alpha$-dependent production of granulocyte-macrophage colony-stimulating factor (GM-CSF) in response to cigarette smoke (CS) exposure is necessary to maintain lipid homeostasis. a) C57BL/6 wild type (WT), IL- $1 \alpha$-deficient and IL- $1 \beta$-deficient mice were exposed to room air (RA) or CS for 4 days. An impact of IL- $1 \alpha$ and IL- $1 \beta$ deficiency was seen on bronchoalveolar lavage fluid (BALF) GM-CSF levels. b-g) BALB/c or C57BL/6 mice were exposed to RA or CS and either GM-CSF-neutralising ( $\alpha \mathrm{GM}$ ) or isotype control (ISO) antibodies, to investigate the impact of GM-CSF neutralisation on b) cell-free BALF cloudiness, c) BALF absorbance at $550 \mathrm{~nm}$, d) BALF phosphatidylcholine levels, CS-induced increase in bronchoalveolar lavage (BAL) e) mononuclear cells and f) neutrophils, and g) BALF IL- $1 \alpha$ levels. At least five animals per experimental group, reproduced at least twice. Ns: nonsignificant. ${ }^{*}$ : not detected. ${ }^{*}: \mathrm{p}<0.05 ;{ }^{* *}: \mathrm{p}<0.01 ;{ }^{* * *}: \mathrm{p}<0.001$.

recruitment and GM-CSF expression were dependent on IL- $1 \alpha$ and partially dependent on IL-1 $\beta$. Finally, we administered hOxLDL intranasally to TLR4-deficient mice. Mirroring the TLR4 dependency of cigarette smoke-induced neutrophilia (fig. 4i) [18, 19], hOxLDL-induced neutrophil recruitment was similarly dependent on TLR4 (fig. 4j). Together, these data show that intranasal delivery of oxidised lipids mimics the main features of cigarette smoke-induced inflammation.

\section{Discussion}

Lung inflammation is a critical component of lung pathologies caused by smoking. Despite remarkable advances in our understanding of cellular and molecular pathways associated with diseases such as COPD, mechanisms by which smoking initiates and propagates inflammatory processes remain incompletely understood. In the present study, we investigated the mechanisms that contribute to the initiation of lung inflammatory processes, with a specific focus on pulmonary lipid homeostasis.

This study was carried out using a well-characterised mouse model of whole-body cigarette smoke exposure $[11,12]$, as well as gene-deficient mice and antibody-based interventions to dissect key molecular mechanisms affected by smoking. In addition to smoke exposure, we pursued proof-of-concept studies using hOxLDL, a rich source of oxidised lipids, to investigate whether changes in lipid homeostasis propagate an inflammatory process reminiscent of cigarette smoke-induced inflammation. 

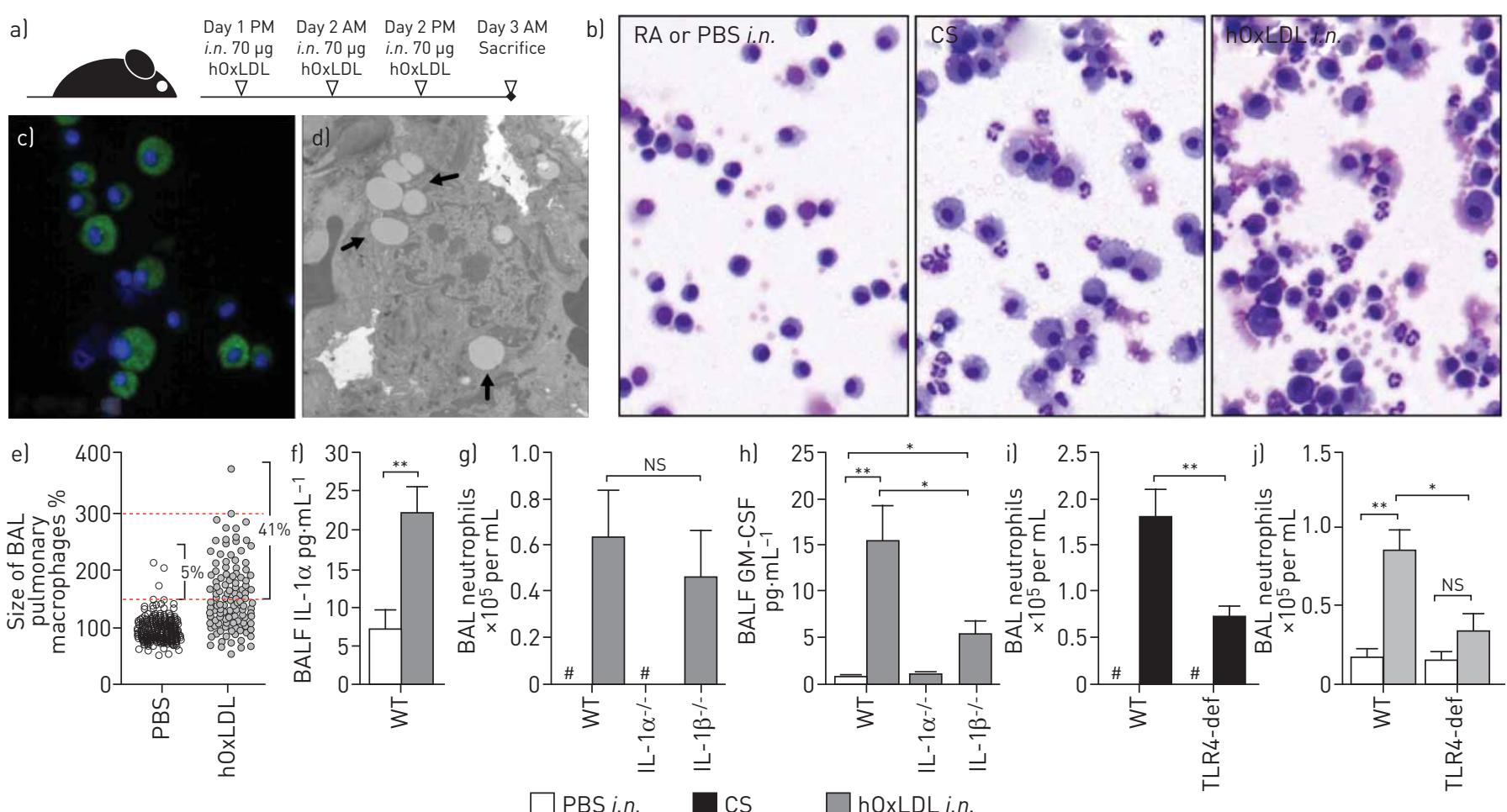

FIGURE 4 Pulmonary exposure to damaged lipids mimics cigarette smoke (CS)-induced inflammation. a) Intranasal (i.n.) delivery protocol of human oxidised low-density lipoprotein (hOxLDL). b) Representative bronchoalveolar lavage (BAL) cytospins from room air (RA)-exposed or PBS-treated, CS-exposed (4 days) and hOxLDL-treated mice. hOxLDL treatment leads to intracellular lipid accumulation in pulmonary macrophages as shown by c) BODIPY (boron-dipyrromethene; green) incorporation (with DAPI (4',6-diamidino-2-phenylindole; blue) staining of nuclei) and d) transmission electron microscopy (arrows show lipid droplets). hOxLDL administration leads to e) an increase in pulmonary macrophage size, f) increased BAL fluid (BALF) interleukin (IL)-1 $\alpha$ levels, g) IL-1 $\alpha$-dependent increase in BAL neutrophils, and h) IL- $1 \alpha$ - and partially IL-1 $\beta$-dependent induction of granulocyte-macrophage colony-stimulating factor (GM-CSF). Impact of Toll-like receptor 4 deficiency (TLR4-def) on both i) CS-induced and j) hOxLDL-induced lung neutrophilia. At least five animals per experimental group, reproduced at least twice. WT: wild type; Ns: nonsignificant. ${ }^{*}$ : not detected. ${ }^{*}: \mathrm{p}<0.05 ;{ }^{* *}: \mathrm{p}<0.01$.

Our study shows for the first time that pulmonary macrophages accumulate lipids within days of cigarette smoke exposure, giving them the appearance of foam cells [13]. The presence of foamy macrophages is a hallmark of cigarette smoke-induced inflammation and is consistently observed following days, weeks and months of smoke exposure. Moreover, lipid accumulation in macrophages persists following smoking cessation. The presence of foamy macrophages in cigarette smoke-exposed mice has been previously documented following chronic exposure in mice [20] and is in agreement with clinical observations made by WILSON et al. [21] in sputum macrophages from smokers. Neither of these observational studies established a link between the presence of foamy macrophages and mechanisms driving lung inflammation.

Lipid accumulation was associated with the release of IL- $1 \alpha$ by pulmonary macrophages. Importantly, we and others previously identified IL- $1 \alpha$ as a key regulator of cigarette smoke-induced inflammation [12, 22], hence linking lipid accumulation in macrophages with cigarette smoke-induced inflammation. Our observations suggest that inflammatory processes associated with cigarette smoke exposure and atherosclerosis may share similar molecular mechanisms. Lipid accumulation in macrophages has been extensively studied in the field of atherosclerosis [13]. It is widely accepted that foam cells drive inflammatory processes in the atherosclerotic plaque [13]. Their formation is a consequence of resident cells failing to handle high levels of oxidised lipids, more specifically oxidised low-density lipoproteins, in the case of atherosclerosis. This initiates an inflammatory cascade resulting in the recruitment of immune inflammatory cells. Therefore, we studied foamy pulmonary macrophages, as they may drive inflammatory processes similar to lipid-laden macrophages observed in atherosclerotic plaques.

In the current study, we provide evidence that IL-1 $\alpha$ plays an important role in maintaining pulmonary lipid homeostasis through a GM-CSF-dependent mechanism following smoke exposure. Specifically, both IL-1 $\alpha$ gene deficiency and antibody-mediated inhibition of GM-CSF led to the accumulation of phospholipids in the BALF following smoke exposure, suggesting an impaired control of surfactant levels. GM-CSF is well known for its role in macrophage differentiation and activation [5]. In fact, impaired GM-CSF signalling is known to be the cause of pulmonary alveolar proteinosis, a disease characterised by 
the accumulation of surfactant in the lungs [5]. Despite the accumulation of phospholipids, we observed no difference in the recruitment of immune cells in anti-GM-CSF-treated animals. This observation is in contrast to previous reports, showing that GM-CSF blockade attenuated inflammatory processes [17, 23]. This is probably a consequence of the dose of antibody used in the current study, which was substantially lower than that previously used to inhibit inflammatory processes. This suggests that, in addition to the previously documented role in neutrophil recruitment [17, 23], GM-CSF activates mechanisms involved in the processing of damaged lipids following smoke exposure.

The source of lipids that accumulate in macrophages is currently not well understood. More than $90 \%$ of surfactant lipids are phospholipids [16] and cigarette smoke's deleterious effects on surfactant has been described as early as the 1960s and 1970s [3,6]. Hence, damaged surfactant lipids are, in our view, the most likely source. Alternatively, membrane lipids from cells that are damaged due to smoke exposure may also accumulate in macrophages. Given the abundance of lipids in surfactant and the critical role pulmonary macrophages play in surfactant turnover, we view cell-derived lipids as a less likely source. Ultimately, determining the definitive source of lipids may not be trivial, as the same molecules (e.g. phosphatidylcholine) are found in surfactant and cell membranes. Aside from the source, our study provides clear evidence for a critical role of lipid accumulation in macrophages in cigarette smoke-induced inflammatory processes. We further show a novel role for IL-1 $\alpha$ in maintaining pulmonary phospholipid homeostasis.

As a proof of concept, we intranasally administered hOxLDL, a rich source of oxidised lipids, to mice. Administration of damaged lipids recapitulated the main cellular and molecular features of cigarette smoke-induced inflammation. Specifically, hOxLDL delivery led to the formation of foamy macrophages and elicited an IL-1 $\alpha$-dependent neutrophilia. These data demonstrate that cigarette smoke and damaged lipids elicit comparable responses. Our findings emphasise the importance of IL-1 $\alpha$ and GM-CSF in the response to a disruption in pulmonary lipid homeostasis. Together, our findings suggest that cigarette smoke's damaging effect on lung surfactant (fig. 5a) causes increased lipid uptake by pulmonary macrophages (fig. 5b). These lipid-laden macrophages release IL-1 $\alpha$ to elicit GM-CSF production, initiating

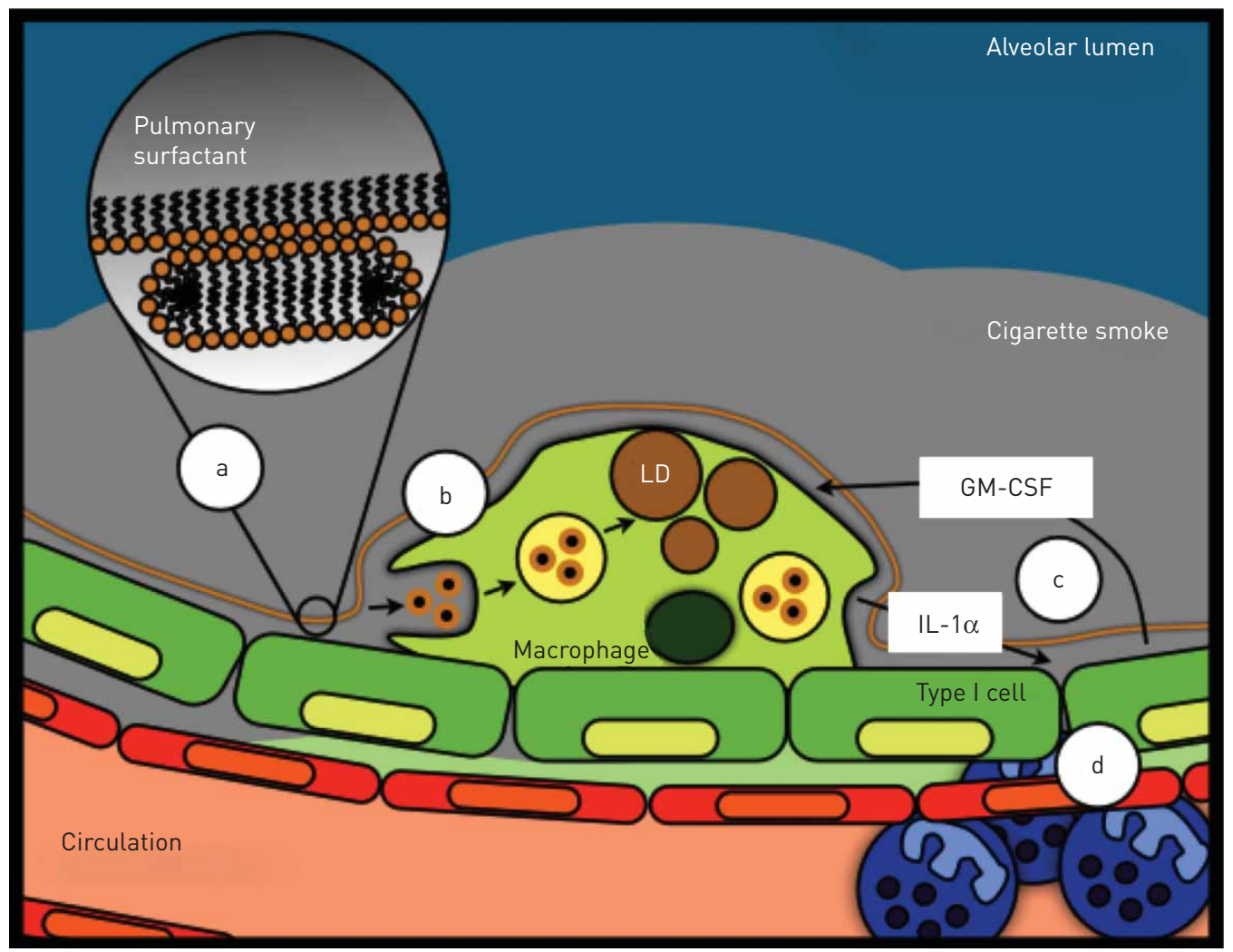

FIGURE 5 Diagram representing the initiation of cigarette smoke-induced inflammation. a) Cigarette smoke damages lung surfactant, which b) causes increased lipid uptake by pulmonary macrophages. c) These lipid-laden macrophages release interleukin (IL)-1 $\alpha$ to elicit granulocyte-macrophage colony-stimulating factor (GM-CSF) production, initiating processes involved in surfactant uptake and degradation. d) IL- $1 \alpha$ also initiates the recruitment of immune inflammatory cells from the circulation, most notably neutrophils. LD: lipid droplets. 
processes involved in surfactant uptake and degradation (fig. 5c). IL-1 $\alpha$ also initiates the recruitment of immune inflammatory cells from the circulation (fig. 5d), most notably neutrophils.

The inflammatory processes described in this manuscript probably represent a protective response to cigarette smoke-induced damage. However, genetic predisposition or environmental factors may precipitate processes that lead to the expression of pathophysiological changes characteristic of COPD and interstitial lung diseases. Of note, we recently reported that activation of signalling pathways associated with lipid metabolism are conserved between human smokers and cigarette smoke-exposed mice [7]. Moreover, exacerbation of cigarette smoke-induced inflammatory processes by nontypeable Haemophilus influenzae is driven by IL-1 $\alpha$ in cigarette smoke-exposed mice [24]. Additional research is required to identify factors that distort the normal/protective response to cigarette smoke and cause chronic lung diseases. Here, we report that impaired pulmonary lipid homeostasis contributes to cigarette smoke-induced inflammation. A better understanding of these mechanisms may guide the design of novel immune-modulatory interventions aimed at restoring lipid homeostasis.

This study delineates a novel mechanism by which cigarette smoke elicits inflammation and highlights the importance of disrupted pulmonary lipid homeostasis in the early pathogenesis of smoking-related diseases. The formation of foam cells in the lungs bears similarities to pathogenic processes associated with atherosclerosis; in both instances, the immune system attempts to maintain lipid homeostasis in their respective compartment. This study provides a novel paradigm for cigarette smoke-induced inflammatory processes, and introduces the opportunity for intervention strategies aimed at regulating lipid homeostasis in the lungs. Further research is required to investigate whether accumulation of lipids in macrophages is a common pathogenic mechanism shared among chronic inflammatory disorders.

\section{Acknowledgements}

The authors would like to sincerely acknowledge the technical help of Joanna Kasinska (Dept of Pathology and Molecular Medicine, McMaster Immunology Research Centre, McMaster University, Hamilton, ON, Canada) and Marcia Reid (Electron Microscopy Facility, Health Science Centre, McMaster University), as well as the administrative support of Marie Bailey (Dept of Pathology and Molecular Medicine, McMaster Immunology Research Centre, McMaster University).

\section{References}

1 US Dept of Health and Human Services. The Health Consequences of Smoking: 50 Years of Progress. A Report of the Surgeon General. Atlanta, US Dept of Health and Human Services, Centers for Disease Control and Prevention, National Center for Chronic Disease Prevention and Health Promotion, Office on Smoking and Health, 2014

2 Martin RR. Altered morphology and increased acid hydrolase content of pulmonary macrophages from cigarette smokers. Am Rev Respir Dis 1973; 107: 596-601.

3 Pratt SA, Finley TN, Smith MH, et al. A comparison of alveolar macrophages and pulmonary surfactant(?) obtained from the lungs of human smokers and nonsmokers by endobronchial lavage. Anat Rec 1969; 163: 497-507.

4 Guilliams M, Lambrecht BN, Hammad H. Division of labor between lung dendritic cells and macrophages in the defense against pulmonary infections. Mucosal Immunol 2013; 6: 464-473.

5 Carey B, Trapnell BC. The molecular basis of pulmonary alveolar proteinosis. Clin Immunol 2010; 135: 223-235.

6 Finley TN, Ladman AJ. Low yield of pulmonary surfactant in cigarette smokers. N Engl J Med 1972; 286: 223-227.

7 Morissette MC, Lamontagne M, Berube JC, et al. Impact of cigarette smoke on the human and mouse lungs: a gene-expression comparison study. PLoS One 2014; 9: e92498.

8 Rahman I, van Schadewijk AA, Crowther AJ, et al. 4-Hydroxy-2-nonenal, a specific lipid peroxidation product, is elevated in lungs of patients with chronic obstructive pulmonary disease. Am J Respir Crit Care Med 2002; 166: $490-495$.

9 Aoshiba K, Koinuma M, Yokohori N, et al. Immunohistochemical evaluation of oxidative stress in murine lungs after cigarette smoke exposure. Inhal Toxicol 2003; 15: 1029-1038.

10 Malhotra D, Thimmulappa R, Navas-Acien A, et al. Decline in NRF2-regulated antioxidants in chronic obstructive pulmonary disease lungs due to loss of its positive regulator, DJ-1. Am J Respir Crit Care Med 2008; 178: 592-604.

11 Botelho FM, Gaschler GJ, Kianpour S, et al. Innate immune processes are sufficient for driving cigarette smoke-induced inflammation in mice. Am J Respir Cell Mol Biol 2010; 42: 394-403.

12 Botelho FM, Bauer CM, Finch D, et al. IL-1 $\alpha / \mathrm{IL}-1 \mathrm{R} 1$ expression in chronic obstructive pulmonary disease and mechanistic relevance to smoke-induced neutrophilia in mice. PLoS One 2011; 6: e28457.

13 Lundberg AM, Hansson GK. Innate immune signals in atherosclerosis. Clin Immunol 2010; 134: 5-24.

14 Moore KJ, Sheedy FJ, Fisher EA. Macrophages in atherosclerosis: a dynamic balance. Nat Rev Immunol 2013; 13: 709-721.

15 Botelho FM, Nikota JK, Bauer CM, et al. Cigarette smoke-induced accumulation of lung dendritic cells is interleukin-1 $\alpha$-dependent in mice. Respir Res 2012; 13: 81.

16 Agassandian M, Mallampalli RK. Surfactant phospholipid metabolism. Biochim Biophys Acta 2013; 1831: 612-625.

17 Vlahos R, Bozinovski S, Chan SP, et al. Neutralizing granulocyte/macrophage colony-stimulating factor inhibits cigarette smoke-induced lung inflammation. Am J Respir Crit Care Med 2010; 182: 34-40.

18 Doz E, Noulin N, Boichot E, et al. Cigarette smoke-induced pulmonary inflammation is TLR4/MyD88 and IL-1R1/MyD88 signaling dependent. J Immunol 2008; 180: 1169-1178. 
19 Maes T, Bracke KR, Vermaelen KY, et al. Murine TLR4 is implicated in cigarette smoke-induced pulmonary inflammation. Int Arch Allergy Immunol 2006; 141: 354-368.

20 Hirama N, Shibata Y, Otake K, et al. Increased surfactant protein-D and foamy macrophages in smoking-induced mouse emphysema. Respirology 2007; 12: 191-201.

21 Wilson AM, Nair P, Hargreave FE, et al. Lipid and smoker's inclusions in sputum macrophages in patients with airway diseases. Respir Med 2011; 105: 1691-1695.

22 Pauwels NS, Bracke KR, Dupont LL, et al. Role of IL-1 $\alpha$ and the Nlrp3/caspase-1/IL-1 $\beta$ axis in cigarette smoke-induced pulmonary inflammation and COPD. Eur Respir J 2011; 38: 1019-1028.

23 Botelho FM, Nikota JK, Bauer C, et al. A mouse GM-CSF receptor antibody attenuates neutrophilia in mice exposed to cigarette smoke. Eur Respir J 2011; 38: 285-294.

24 Nikota JK, Shen P, Morissette MC, et al. Cigarette smoke primes the pulmonary environment to IL-1 $\alpha /$ CXCR-2-dependent nontypeable Haemophilus influenzae-exacerbated neutrophilia in mice. J Immunol 2014; 193: 3134-3145. 